\title{
A Variational Framework for Joint Segmentation and Registration
}

\author{
Anthony Yezzi*, Lilla Zöllei ${ }^{\dagger}$, and Tina Kapur ${ }^{\ddagger}$ \\ ${ }^{*}$ School of Electrical and Computer Engineering, Georgia Institute of Technology, Atlanta, GA \\ ${ }^{\dagger}$ Artificial Intelligence Laboratory, Massachusetts Institute of Technology, Cambridge, MA \\ ${ }_{\ddagger}$ Visualization Technology, Inc., Lawrence, MA
}

\begin{abstract}
Traditionally, segmentation and registration have been solved as two independent problems, even though it is often the case that the solution to one impacts the solution to the other. In this paper, we introduce a geometric, variational framework that uses active contours to simultaneously segment and register features from multiple images. The key observation is that multiple images may be segmented by evolving a single contour as well as the mappings of that contour into each image. To the best of our knowledge, this is the first attempt at interleaving segmentation and registration in such a framework.
\end{abstract}

\section{Introduction}

Segmentation and registration have been established as important problems in the field of medical image analysis $[1,8,12,26]$. Traditionally, solutions have been developed for each of these two problems in relative isolation from the other, but with increasing dependence on the existence of a solution for the other. In the rest of this section, we discuss the interdependence of segmentation and registration solutions and introduce our motivation for a method that simultaneously estimates the two.

Dependence of Registration on Segmentation: A large class of registration solutions, referred to as "feature-based" methods, require that some features be identified or segmented in the images prior to their registration. These features may be identified using low-level methods such as edge-detection, or segmented using higher level methods that are customized for specific anatomical structures. In contrast to feature-based registration methods, a second class of methods, referred to as "intensity-based" segmentation methods, require no apriori segmentation, which makes them an attractive proposition. Mutual-Information was introduced as a particularly effective intensity-based metric for registration of medical imagery [10, 27], and it's applicability has been repeatedly demonstrated for solving rigid (6 degree of freedom) registration problems. No such consensus, feature-based or intensity-based, seems to have been reached for the domain of non-rigid registration.
Dependence of Segmentation on Registration: The dependence of segmentation on registration is somewhat more subtle. A large class of segmentation methods do not depend on explicit registration between multiple data sets. We will refer to these as "low-level" segmentation methods. In these low-level segmentation methods, the algorithm designers typically use information synthesized from their knowledge of several example data sets to set the parameters of their segmentation algorithms, but no explicit process of registering those data sets to a common reference frame is carried out prior to segmentation. These methods may process a single channel input image using image-processing techniques such as thresholding, connectivity analysis, region-growing, morphology, snakes, and Bayesian MAP estimation. Or, they may process multichannel data in which the channels are naturally registered because they are acquired simultaneously.

While it is easier to get started in segmentation using these methods because there is no need to solve the cumbersome registration problem apriori, efforts in low-level segmentation of medical imagery often conclude that "modelbased", higher level information such as the shape, appearance, and relative geometry of anatomy need to be incorporated into the solution in order to complete the segmentation task $[23,11,24,13]$. And it is in the building of these models of anatomy that registration plays a key role; Individual data sets need to be registered to a common frame of reference, so that statistics about their shape, appearance, or relative geometry can be gathered.

The work presented in this paper is motivated by the desire to interleave the process of segmentation and registration so that both solutions may be built simultaneously and hence to eliminate the need to completely deliver one solution before being able to start the other. This challenge has been approached with a min-max entropy based framework to segment and register portal images to CT [2].

The focus of this paper is to introduce a geometric, variational, active contour, framework that allows us to interleave powerful level-set based formulations of segmentation with a feature based registration method. To the best of our knowledge, this is the first attempt at interleaving segmentation and registration in a geometric, variational formulation. 


\section{Background on Active Contours}

Active contours have been utilized extensively for problems including image segmentation, visual tracking, and shape analysis (see [3] and the references therein). A variety of active contour models have been proposed since the introduction of the "snake" methodology in the mid-1980's [14]. These original models utilized parametric representations of the evolving contour. Shortly thereafter, using the level set methodology of Osher and Sethian [18], more geometric techniques (such as those presented in [15]) began to arise based upon the theory of curve evolution. An important class of these geometric models were derived via the Calculus of Variations to obtain evolution equations which would minimize energy functionals (or "objective functions") tailored to features of interest in the image data. An in-depth discussion of variational image segmentation methods, as well an extensive list of references, may be found in the book [16]. The model that will be presented in this paper certainly fits within the context of these geometric variational approaches. However, we will exploit the calculus of variations to address not only the problem of image segmentation, but simultaneously the problem of image registration as well.

Most of the early active contour models for image segmentation, such as $[4,5,9,14,15,25,28]$, were designed to capture localized image features, most notably edges. As such, these have come to be known as "edge-based" models. In medical imaging and many other important applications where consistently strong edge information is not always present along the entire boundary of the objects to be segmented, the performance of purely edge-based models is often inadequate. In recent years, a large class of region based models (such as $[6,7,19,21,22,29]$ ) have utilized image information not only near the evolving contour but also image statistics inside and outside the contour (in many ways inspired by the "Region Competition" algorithm presented by Zhu and Yuille [30]) in order to improve the performance.

There are still many cases in which both edge and region based active contour models have difficulty yielding correct segmentations of images which present rather subtle information about portions of the object to be captured. Significant improvement may be obtained in such cases by combining information from images of the same object acquired using different modalities (CT and MR, for example). However, to utilize the joint information, the various images must be correctly aligned to each other or "registered." If this can be done prior to segmenting any of the images, then registration can assist segmentation.

It is equally true, on the other hand, that segmentation can assist registration. It is typically much easier to align two images if the boundary of a common object or some other set of common point features have have been accu- rately detected in both images beforehand. The images may then be registered by point feature or contour matching. Furthermore, there may be cases in which registration is impossible (at least rigid registration) without some level of segmentation. This is the case when two (or more) images contain multiple common objects which may not be related by a single global mapping between the image domains. For example, an x-ray image of the femur and tibia may not be globally registered to a CT image of the femur and tibia if the knee is bent differently in the two images. Yet it is certainly possible to choose a registration which aligns the two femoral bones or a different registration which aligns the two tibial bones. In either case, though, it is necessary to segment the desired object from both images in order to perform the registration.

Next, we outline a geometric, variational framework for simultaneously segmenting and registering common objects in two or more images (the technical discussion will consider just two images, but the approach is easily adapted to multiple images). While our methodology is quite general and may certainly utilize any number of segmentation energy functionals, we focus our attention around region based energy functionals; in particular, we will utilize the piecewise constant Mumford-Shah [17] energy presented in [7].

\section{General Framework}

In this section we outline the general framework for joint registration and segmentation via active contours. Later, in Section 4 we will address rigid registration as a special case. Our model will be derived first for the two dimensional case, and then the corresponding three dimensional active surface model will be presented. We begin by establishing some basic notation.

\subsection{Notation and Problem Statement}

Let $I: \Omega \subset \Re^{2} \rightarrow \Re$ and $\hat{I}: \hat{\Omega} \subset \Re^{2} \rightarrow \Re$ denote two images that contain a common object to be registered and segmented, and let $g: \Re^{2} \rightarrow \Re^{2}$ be an element of a finite dimensional group $G$ with parameters $g_{1}, \ldots, g_{n}$. We will denote by $\hat{\mathbf{x}} \in \hat{\Omega}$ the image of a point $\mathbf{x} \in \Omega$ under $g$ (i.e. $\hat{\mathbf{x}}=g(\mathbf{x})$ ), and we will denote the Jacobian matrix of $g$ by $g^{\prime}$ and its determinant (which we assume is positive) by $\left|g^{\prime}\right|$.

Our goal may be stated as follows. We wish to find a closed curve $C \subset \Omega$ which captures the boundary of an object in image $I$, and another closed curve $\hat{C} \subset \hat{\Omega}$ which captures the boundary of the corresponding object in image $\hat{I}$. If $C$ and $\hat{C}$ were independent, this would simply be a segmentation problem. However, we will relate $C$ and $\hat{C}$ through a mapping $g \in G$.

$$
\hat{C}:=g(C)
$$


Our problem, then, is to find both a mapping $g$ (which we will refer to from now on as the registration) and a contour $C$ such that $C$ and $\hat{C}=g(C)$ yield desirable segmentations of $I$ and $\hat{I}$ respectively. In this manner, the segmentation and registration problems become coupled very naturally.

We will make use of the following additional notation. $T, N$ and $\hat{T}, \hat{N}$ will denote the unit tangents and normals to $C$ and $\hat{C}$ respectively. In the same manner, $d \hat{\mathbf{x}}$ will denote the area measure $d \mathbf{x}$ (of $\Omega$ ) pushed forward (onto $\hat{\Omega}$ ) by $g$, and $d \hat{s}$ will denote the arc length measure $d s$ (of $C$ ) pushed forward (onto $\hat{C}$ ) by $g$. The relationships between these measures are given by $d \hat{\mathbf{x}}=\left|g^{\prime}\right| d \mathbf{x}$ and by $d \hat{s}=\left\|g^{\prime} T\right\| d s$. Finally, let $C_{\text {in }} \subset \Omega$ and $C_{\text {out }} \subset \Omega$ denote the regions inside and outside the curve $C$ and let $\hat{C}_{\text {in }} \subset \hat{\Omega}$ and $\hat{C}_{\text {out }} \subset$ $\hat{\Omega}$ denote the regions inside and outside the curve $\hat{C}$.

\subsection{Energy Functional}

If we were charged with the task of segmenting image $I$ and $\hat{I}$ separately (i.e. without enforcing a relationship between $C$ and $\hat{C}$ ), then we might choose from any number of geometric energy based active contour models and would certainly be free to utilize two different models if the characteristics of image $I$ and $\hat{I}$ were sufficiently different. Let us refer to the energy functionals associated with these two models as $E_{1}$ and $E_{2}$ respectively.

In order to discuss the problem in more detail, we must choose a specific form for $E_{1}$ and $E_{2}$. Because of their wider capture range and greater robustness to noise, we prefer to focus our discussion around region based energy functionals rather than edge based energy functions; although, a similar development can be followed for just about any class of geometric active contour energies (even more sophisticated models that incorporate both edge and region measurements, shape priors, anatomical constraints, and other considerations).

A general class of region-based energies exhibit the following form,

$$
\begin{aligned}
& E_{1}(C)=\int_{C_{\text {in }}} f_{\text {in }}(\mathbf{x}) d \mathbf{x}+\int_{C_{\text {out }}} f_{\text {out }}(\mathbf{x}) d \mathbf{x} \\
& E_{2}(\hat{C})=\int_{\hat{C}_{\text {in }}} \hat{f}_{\text {in }}(\mathbf{x}) d \mathbf{x}+\int_{\hat{C}_{\text {out }}} \hat{f}_{\text {out }}(\mathbf{x}) d \mathbf{x}
\end{aligned}
$$

where the integrands $f_{\text {in }}$ and $f_{\text {out }}$ depend on $I$ and where the integrands $\hat{f}_{\text {in }}$ and $\hat{f}_{\text {out }}$ depend on $\hat{I}$. If we introduce an artificial time variable we obtain the following gradient evolutions for $C$ and $\hat{C}$.

$$
\begin{aligned}
& \frac{\partial C}{\partial t}=\left(f_{\text {in }}-f_{\text {out }}\right) N \\
& \frac{\partial \hat{C}}{\partial t}=\left(\hat{f}_{\text {in }}-\hat{f}_{\text {out }}\right) \hat{N}
\end{aligned}
$$

For example, the piecewise-constant segmentation model of Chan and Vese [7], which the authors utilized for the experiments in this paper, favors a curve which yields the least total squared error approximation of the image by one constant inside the curve and another constant outside the curve. This yields the following particular choices for $f_{\text {in }}, f_{\text {out }}, \hat{f}_{\text {in }}$, and $\hat{f}_{\text {out }}$,

$$
\begin{array}{ll}
f_{\text {in }}=(I-u)^{2} & f_{\text {out }}=(I-v)^{2} \\
\hat{f}_{\text {in }}=(\hat{I}-\hat{u})^{2} & \hat{f}_{\text {out }}=(\hat{I}-\hat{v})^{2}
\end{array}
$$

where $u$ and $v$ denote the mean values of $I$ inside and outside $C$ and where $\hat{u}$ and $\hat{v}$ denote the mean values of $\hat{I}$ inside and outside $\hat{C}$.

By combining the selected energy functionals and enforcing the relationship $\hat{C}=g(C)$, we may formulate a joint energy that depends on $g$ and $C$.

$$
\begin{aligned}
& E(g, C)=E_{1}(C)+E_{2}(g(C))= \\
& \quad \int_{C_{\text {in }}} f_{\text {in }}(\mathbf{x}) d \mathbf{x}+\int_{C_{\text {out }}} f_{\text {out }}(\mathbf{x}) d \mathbf{x}+\int_{\hat{C}_{\text {in }}} \hat{f}_{\text {in }}(\mathbf{x}) d \mathbf{x}+\int_{\hat{C}_{\text {out }}} \hat{f}_{\text {out }}(\mathbf{x}) d \mathbf{x}
\end{aligned}
$$

We may re-express this energy using integrals only over the space $\Omega$, which contains the contour $C$, as follows.

$$
\begin{aligned}
& E(g, C)= \\
& \quad \int_{C_{\text {in }}}\left(f_{\text {in }}+\left|g^{\prime}\right| \hat{f}_{\text {in }} \circ g\right)(\mathbf{x}) d \mathbf{x}+\int_{C_{\text {out }}}\left(f_{\text {out }}+\left|g^{\prime}\right| \hat{f}_{\text {out }} \circ g\right)(\mathbf{x}) d \mathbf{x}
\end{aligned}
$$

Now that task is to choose $g$ and $C$ in order to minimize (6). In doing so, we simultaneously segment both $I$ and $\hat{I}$ via $C$ and $\hat{C}$ as well as register the detected features (which are guaranteed to have the same detected shape since the contours $C$ and $\hat{C}$ will not be deformed independently) to each other through the mapping $g$.

Remarks: Obviously a weighted combination of $E_{1}$ and $E_{2}$ would be more general and useful in the event that one image is easier to segment than the other. However, to keep the development as clean and simple as possible, we will not include such weights (we will follow a similar convention of ignoring weighting coefficients when we add curvature terms to the upcoming gradient flows). A more significant point, though, is that (6) does not allow the registration $g$ to be directly influenced by $E_{1}$. This is a result of our arbitrary choice to let the unknown curve $C$ live in the domain $\Omega$ of image $I$. A more symmetric arrangement would involve utilizing a separate domain for $C$ and two mappings $g_{1} \in G$ and $g_{2} \in G$ to map $C$ into $\Omega$ and $\hat{\Omega}$ respectively. Then the actual registration between the $\Omega$ and $\hat{\Omega}$ would be given by $g_{2} \circ g_{1}^{-1}$. Once again, we have chosen to keep the presentation as simple as possible by considering only one mapping $g$, which requires us to arbitrarily place the unknown curve in one of the two image domains. 


\subsection{Gradient Flows}

The most straightforward method for minimizing $E(C, g)$ is to start with an initial guess for both $C$ and $g$ and then evolve the contour $C$ and the registration parameters $g_{1}, g_{2}, \ldots, g_{n}$ using a gradient flow.

The gradient evolution for the curve $C$ may be obtained immediately by noticing that (6) has the same form as (2). Thus, its gradient flow has the same form as (4). Simple substitution yields

$$
\begin{aligned}
& \frac{\partial C}{\partial t}=\left(f+\left|g^{\prime}\right| \hat{f} \circ g\right) N \\
& \text { where } f=f_{\text {in }}-f_{\text {out }} \text { and } \hat{f}=\hat{f}_{\text {in }}-\hat{f}_{\text {out }} \text {. }
\end{aligned}
$$

This flow, by itself, however, is not guaranteed to keep the evolving curve smooth. Thus, as is standard in most geometric active contour models we will add a curvature $(\kappa)$ term to the gradient flow (which arises if we add an arc length penalty to our energy functional) in order to regularize the curve evolution.

$$
\frac{\partial C}{\partial t}=\left(f+\left|g^{\prime}\right| \hat{f} \circ g\right) N-\kappa N
$$

The gradient evolutions for the registration parameters $g_{1}, \ldots, g_{n}$ depend upon the geometry of the curve $\hat{C}$ and are given by

$$
\begin{aligned}
\frac{d g_{i}}{d t} & =\frac{\partial E}{\partial g_{i}} \\
& =\int_{\hat{C}}\left\langle\frac{\partial \hat{\mathbf{x}}}{\partial g_{i}}, \hat{f}(\hat{\mathbf{x}}) \hat{N}\right\rangle d \hat{s} \\
& =\int_{C}\left\langle\frac{\partial}{\partial g_{i}} g(\mathbf{x}), \hat{f}(g(\mathbf{x})) \hat{N}\right\rangle\left\|g^{\prime} T\right\| d s \\
& =\int_{C}\left\langle\frac{\partial}{\partial g_{i}} g(\mathbf{x}), \hat{f}(g(\mathbf{x})) J\left(g^{\prime} T\right)\right\rangle d s
\end{aligned}
$$

(the last step uses the fact that $\hat{N}=J\left(\frac{g^{\prime} T}{\left\|g^{\prime} T\right\|}\right)$ where $J$ denotes the ninety degree rotation matrix).

\section{Rigid Registration}

Notice that the gradient curve evolution (8) for $C$ and the gradient direction (9) for the vector of registration parameters $g_{1}, \ldots, g_{n}$ both depend upon the Jacobian, $g^{\prime}$ of the registration map $g$. In the special case where $G$ is the group of rigid motions, then we may represent $g$ by a rotation matrix $R$ and a translation vector $T$.

$$
g(\mathbf{x})=R \mathbf{x}+T
$$

In this case, the Jacobian of $g$ is independent of $\mathbf{x}$ and is simply the rotation matrix $R$, which has a unit determinant, thereby greatly simplifying both (8) and (9).

$$
\begin{aligned}
\frac{\partial C}{\partial t} & =(f(\mathbf{x})+\hat{f}(g(\mathbf{x}))) N-\kappa N \\
\frac{d g_{i}}{d t} & =\int_{C} \hat{f}(g(\mathbf{x}))\left\langle\frac{\partial g(\mathbf{x})}{\partial g_{i}}, \hat{N}\right\rangle d s
\end{aligned}
$$

\subsection{The 2D case}

In two dimensions, the rotation matrix $R$ depends upon a single angle $\theta$ and the translation vector $T$ depends upon two offsets $T_{x}$ and $T_{y}$ in the $x$ and $y$ directions respectively.

$$
R=\left[\begin{array}{rr}
\cos \theta & \sin \theta \\
-\sin \theta & \cos \theta
\end{array}\right], \quad T=\left[\begin{array}{c}
T_{x} \\
T_{y}
\end{array}\right]
$$

The partial derivatives of $g(\mathbf{x})$, needed in (12) with respect to these three registration parameters are given by

$$
\begin{gathered}
\frac{\partial g(\mathbf{x})}{\partial \theta}=\left[\begin{array}{rr}
-\sin \theta & \cos \theta \\
-\cos \theta & -\sin \theta
\end{array}\right] *\left[\begin{array}{l}
x \\
y
\end{array}\right], \\
\frac{\partial g(\mathbf{x})}{\partial T_{x}}=\left[\begin{array}{l}
1 \\
0
\end{array}\right], \quad \frac{\partial g(\mathbf{x})}{\partial T_{y}}=\left[\begin{array}{l}
0 \\
1
\end{array}\right] .
\end{gathered}
$$

\subsection{The 3D case}

In three dimensions, we evolve a surface $S$ rather than a curve $C$ and our registration $g$ now represents a mapping from $R^{3}$ to $R^{3}$. However, for the case of rigid registration, $g$ still has the form of (10). The rotation matrix $R$ can be represented by a product of three separate rotation matrices $R_{\alpha}, R_{\beta}$, and $R_{\gamma}$ which cause rotations around the $x, y$, and $z$ axis respectively. We refer to the corresponding angles as roll $(\alpha)$, pitch $(\beta)$, and yaw $(\gamma)$. The translation vector $T$ depends upon three offsets $T_{x}, T_{y}$, and $T_{z}$ in the $x, y$, and $z$ directions respectively.

$$
\begin{array}{r}
R=R_{\gamma} * R_{\beta} * R_{\alpha}, \quad T=\left[\begin{array}{l}
T_{x} \\
T_{y} \\
T_{z}
\end{array}\right] \\
R_{\alpha}=\left[\begin{array}{rrr}
1 & 0 & 0 \\
0 & \cos \alpha & -\sin \alpha \\
0 & \sin \alpha & \cos \alpha
\end{array}\right] \\
R_{\beta}=\left[\begin{array}{rrr}
\cos \beta & 0 & \sin \beta \\
0 & 1 & 0 \\
-\sin \beta & 0 & \cos \gamma
\end{array}\right] \\
R_{\gamma}=\left[\begin{array}{rrr}
\cos \gamma & -\sin \gamma & 0 \\
\sin \gamma & \cos \gamma & 0 \\
0 & 0 & 1
\end{array}\right]
\end{array}
$$

The partial derivatives of $g(\mathbf{x})$ with respect to these six registration parameters are given by

$$
\frac{\partial g(\mathbf{x})}{\partial \alpha}=R_{\gamma} R_{\beta} R_{\alpha}^{\prime} \mathbf{x}+T
$$




$$
\begin{gathered}
\frac{\partial g(\mathbf{x})}{\partial \beta}=R_{\gamma} R_{\beta}^{\prime} R_{\alpha} \mathbf{x}+T \\
\frac{\partial g(\mathbf{x})}{\partial \gamma}=R_{\gamma}^{\prime} R_{\beta} R_{\alpha} \mathbf{x}+T \\
\frac{\partial g(\mathbf{x})}{\partial T_{x}}=\left[\begin{array}{l}
1 \\
0 \\
0
\end{array}\right], \quad \frac{\partial g(\mathbf{x})}{\partial T_{y}}=\left[\begin{array}{l}
0 \\
1 \\
0
\end{array}\right], \quad \frac{\partial g(\mathbf{x})}{\partial T_{z}}=\left[\begin{array}{l}
0 \\
0 \\
1
\end{array}\right] .
\end{gathered}
$$

These derivatives are utilized to update the registration parameters via the coupled flow for the surface $S$ and the registration $g$ according to the following equations (analogous to (11) and (12) for the 2D case)

$$
\begin{aligned}
\frac{\partial S}{\partial t} & =(f(\mathbf{x})+\hat{f}(g(\mathbf{x}))) N-H N \\
\frac{d g_{i}}{d t} & =\int_{S} \hat{f}(g(\mathbf{x}))\left\langle\frac{\partial g(\mathbf{x})}{\partial g_{i}}, \hat{N}\right\rangle d A,
\end{aligned}
$$

where $H$ and $d A$ denote the mean curvature and area element of the surface $S$ ( $N$ and $\hat{N}$ denote the unit normal of $S$ and $\hat{S}=g(S)$ just as in the 2D case for curves).

\section{Results}

In this section, we report segmentation/registration results from three experiments on MRI/CT images of the head and the spine. The first two experiments were performed in $2 \mathrm{D}$, while the third one was performed in 3D. In the $2 \mathrm{D}$ experiments, corresponding slices between the MR and the CT were chosen manually, and used as input for our algorithm. In the 3D experiment, a pair of 3D MR and CT scans was used as input, without any attempt to manually initialize the registration. In all three cases, validation is currently performed by visual inspection of the results.

\subsection{D MR-CT Head Experiment}

Input: In this experiment, the input consists of two 2D images of the head. The first input image is a single, axial, cross-section from a 3D, gradient echo MRI scan (top row of Figure 1), and the second image is the (manually chosen) corresponding cross-section from a 3D CT scan (bottom row of Figure 1).

Goal: The goal of the joint segmentation-registration experiment is to simultaneously segment the skin surface and register the two slices.

Initialization: A curve is initialized on the MR image, such that it lies within the head images. This initial curve is shown in red in the left column of Figure 1. We choose initial registration parameters $\left(T_{x} ; T_{y} ; \theta\right)=(0,0,0)$ which map this initial curve inside the head of the CT image but clearly not at the "corresponding location."

Outcome: The final joint segmentation is shown in the right column of Figure 1. Notice that the red contour accurately outlines the skin surface in both the MR and CT images. Convergence was achieved in 450 steps. At convergence, the registration was reported as $\left(T_{x} ; T_{y} ; \theta\right)=$ $7.26 ; 6.1 ;-0.807$. Observe that as the contour evolves (left to right) within the MR image (top), its rigid transformation into the CT image (bottom) is also evolving.

\subsection{D MR-CT Spine Experiment}

Input: In this experiment, the input consists of two 2D images of the spine. The first input image is a single, sagittal, cross-section from a 3D, MRI scan (left column of Figure 2 ), and the second image is the (manually chosen) corresponding cross-section from a 3D CT scan (right column).

Goal: The goal of the joint segmentation-registration experiment is to segment a single vertebra (bright in MR, brighter in CT) while computing the rigid transform that registers the two vertebra in the different images. Note that the two spine images can't be registered by a single rigid transform.

Initialization: A curve is initialized within a vertebra in the MR image (shown in red in the first row of Figure 2). The initial registration parameters $\left(T_{x} ; T_{y} ; \theta\right)=(0,0,0)$ map this initial curve into the same vertebra in the CT image, but not exactly to the corresponding portion of the vertebra. Outcome: The segmentation component of the result is shown in the last row of Figure 2. Notice that the red contour accurately outlines the boundary of the vertebra in each of the MR and the CT images. By segmenting/registering each vertebra in this manner, the change in the curvature of the spine can be estimated between two scans.

\subsection{D CT-MR Head Experiment}

Input: In this experiment, the input consists of two 3D data sets of the head, one MR, one CT. Each data set contains 23 slices of size $256 x 256$. The middle row of Figure 3 shows the an axial slice from the MR data set, and the bottom row shows the corresponding slice from the CT data set.

Goal: The goal of the joint segmentation-registration experiment is to segment the 3D skin surface while registering the two 3D data sets.

Initialization: A surface is initialized such that it lies outside the head in the MR image. This initial surface is shown in the top left corner of Figure 3. Initial registration parameters $\left(T_{x} ; T_{y} ; \theta\right)$ are all set to be 0 .

Outcome: The segmentation component of the result is shown in different forms in each of the three rows of Figure 3. The first column of the top row shows the initial 3D surface, the second column of the first row shows an intermediate configuration of the surface during evolution, and the right column shows the surface at convergence (100 iterations). The second and third rows show cross-sections of the surface overlaid on axial MR and CT slices, respectively. In each row, the first column shows the initial surface intersected with the MR/CT slice, the second column shows 
an intermediate state of the surface, and the third column shows the final state of the surface intersected with the two slices.

\section{Summary and Future Work}

We have presented a variational framework for joint segmentation and registration using active contours. We employ a single contour (or surface in $3 \mathrm{D}$ ) to segment multiple images. The contour and the registration are both computed to minimize a set of energy functionals, one for each image. The experiments in this paper utilize an intensity-based energy functional, but the framework allows for richer choices that may encode shape priors, textures, or other image statistics, which we are currently exploring.

\section{References}

[1] N. Ayache "Medical Computer Vision, Virtual Reality and Robotics," Image and Vision Computing p 295-313; 1995

[2] R. Bansal, L.H. Staib, Z. Chen, A. Rangarajan, J. Knisely, R. Nath and J. Duncan "Entropy-Based, Multiple-Portal-to-3D CT Registration for Prostate Radiotherapy Using Iteratively Estimated Segmentation" Proceedings of MICCAI p 567-578; 1999

[3] A. Blake and M. Isard, Active Contours, Springer-Verlag, London 1998.

[4] V. Caselles, F. Catte, T. Coll, and F. Dibos, "A geometric model for active contours in image processing," Numerische Mathematik, vol. 66, pp. 1-31, 1993.

[5] V. Caselles, R. Kimmel, and G. Sapiro, "Geodesic snakes," Int. J. Computer Vision, 1998.

[6] A. Chakraborty, L. Staib, and J. Duncan, "Deformable Boundary Finding in Medical Images by Integrating Gradient and Region Information," IEEE Trans. Medical Imaging, vol. 15, no. 6, pp. 859870, Dec. 1996.

[7] T. Chan and L. Vese, "An active contour model without edges," Int. Conf. Scale-Space Theories in Computer Vision, pp. 141-151, 1999.

[8] H. Cline, W. Lorensen, R. Kikinis, and R. Kikinis "ThreeDimensional Segmentation of MR Images of the Head Using Probability and Connectivity", Journal of Computer-Aided Tomography, vol. 14, no. 6, p 1037-1045; 1990

[9] L. Cohen, "On active contour models and balloons," CVGIP: Image Understanding, vol. 53, pp. 211-218, 1991.

[10] A. Collignon and others, "Automated Multi-Modality Image Registration Based On Information Theory", Proceedings of the Information Processing in Medical Imaging Conference, pp. 263-274, 1995.

[11] T.F. Cootes, A. Hill, C.J. Taylor, and J. Haslam, "Use Of Active Shape Models For Locating Structure In Medical Images", Image and Vision Computing, vol. 12, no. 6, pp. 355-365, 1994.

[12] W.E.L. Grimson, T. Lozano-Perez, W.M. Wells III, G. J. Ettinger, and S. J. White "An Automatic Registration Method for Frameless Stereotaxy, Image, Guided Surgery and Enhanced Reality Visualization”, Proceedings of CVPR, pp. 430-436, 1994.
[13] T. Kapur, W.E.L. Grimson, R. Kikinis, and W.M. Wells III, "Enhanced Spatial Priors for Segmentation of Magnetic Resonance Imagery", Proceedings of Medical Image Computing and Computer Assisted Intervention (MICCAI), pp. 457-468, 1998.

[14] M. Kass, A. Witkin, and D. Terzopoulos, "Snakes: active contour models," Int. Journal of Computer Vision, vol. 1, pp. 321-331, 1987.

[15] R. Malladi, J. Sethian, and B. Vemuri, "Shape modeling with front propagation: a level set approach," IEEE Trans. Pattern Anal. Machine Intell., vol. 17, pp. 158-175, 1995.

[16] J. M. Morel and S. Solimini, Variational Methods in Image Segmentation, Birkhauser, Boston, 1995.

[17] D. Mumford and J. Shah, "Optimal approximations by piecewise smooth functions and associated variational problems," Comm. Pure and App. Mathematics, vol. 42:4, 1989.

[18] S. Osher and J. Sethian, "Fronts propagation with curvature dependent speed: Algorithms based on Hamilton-Jacobi formulations," Journal of Computational Physics, vol. 79, pp. 12-49, 1988.

[19] N. Paragios and R. Deriche, "Geodesic Active Regions for Supervised Texture Segmentation," Proceedings of ICCV, Sept. 1999, Corfu, Greece.

[20] G.P. Penney, J. Weese, J.A. Little, P. Desmedt, D.L.G. Hill, D.J. Hawkes "A Comparison of Similarity Measures for Use in 2D-3D Medical Image Registration" IEEE Transactions on Medical Imaging Vol. 17(4), pp. 586-595, 1998.

[21] R. Ronfard, "Region-Based Strategies for Active Contour Models," Int. J. Computer Vision, vol. 13, no. 2, pp. 229-251, 1994.

[22] C. Samson, L. Blanc-Feraud, G. Aubert, and J. Zerubia. "A Level Set Method for Image Classification," Int. Conf. Scale-Space Theories in Computer Vision, pp. 306-317, 1999.

[23] , L.H. Staib, and J.S. Duncan, "Boundary Finding with Parametrically Deformable Models", PAMI, vol. 14, no. 11, pp. 1061-1075, 1992.

[24] G. Szekely, A. Kelemen, C. Brechbuehler, and G. Gerig, "Segmentation of 3D Objects from MRI Volume Data Using Constrained Elastic Deformations of Flexible Fourier Surface Models", Proceedings of CVRMed95, 1995.

[25] H. Tek and B. Kimia, "Image segmentation by reaction diffusion bubbles," Proc. Int. Conf. Computer Vision, pp. 156-162, 1995.

[26] M. Vannier, R. Butterfield, D. Jordan, W. Murphy and others, "Multi-Spectral Analysis of Magnetic Resonance Images", Radiology, vol. 154, pp. 221-224, 1985

[27] W. Wells, P. Viola, and R. Kikinis, "Multi-Modal Volume Registration by Maximization of Mutual Information", Proceedings of the Second International Symposium on Medical Robotics and Computer Assisted Surgery, pp. 55-62, 1995.

[28] A. Yezzi, S. Kichenassamy, A. Kumar, P. Olver, and A. Tannenbaum, "A Geometric Snake Model for Segmentation of Medical Imagery”, IEEE Trans. Medical Imaging, vol. 16, no. 2, pp. 199-209, 1997.

[29] A. Yezzi, A. Tsai, and A. Willsky, "A Statistical Approach to Image Segmentation for Bimodal and Trimodal Imagery," Proceedings of ICCV, September, 1999.

[30] S. Zhu and A. Yuille, "Region Competition: Unifying snakes, Region Growing, and Bayes/MDL for Multiband Image Segmentation," IEEE Transactions on Pattern Analysis and Machine Intelligence, vol. 18, no. 9, pp. 884-900, Sep. 1996. 
Figure 1: Registration/Segmentation of MR/CT data: Initial (left), intermediate (middle), and final (right) results. The top row shows the evolving contour overlaid on the MR slice, and the bottom row shows it overlaid on the CT slice. Note that the rightmost column shows that the contour has correctly identified the skin boundary in both the CT and the MR.
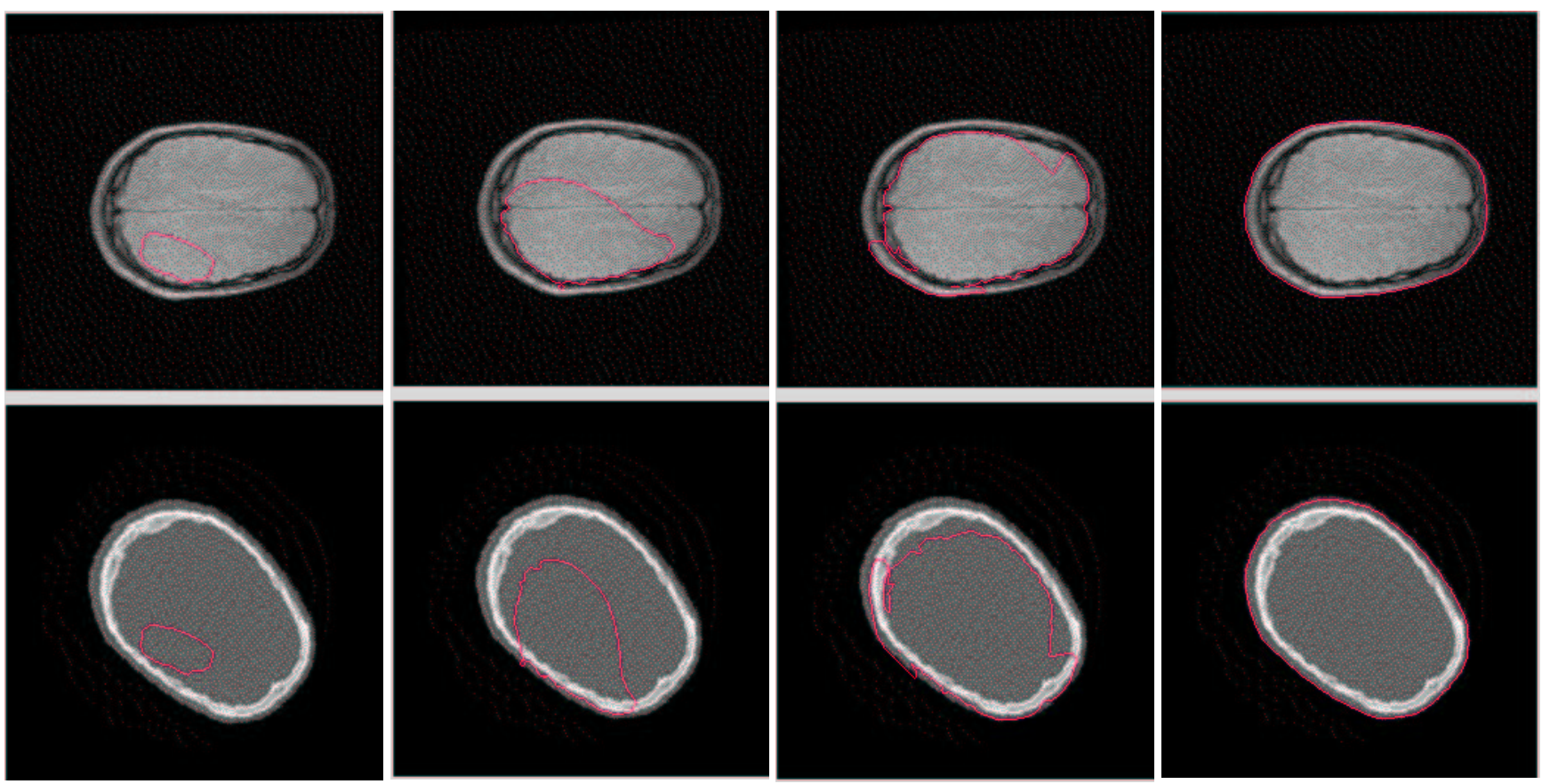

Figure 2: Registration/Segmentation of MR and CT Spine Images: Initial (top) and final (bottom) result. The left column shows $\mathrm{CT}$ images of the spine, and the right column shows MR images. The top row shows the initial contour, placed inside a vertebra, overlaid on both the MR and the CT. Note the poor contrast around the vertebra of interest in the CT image, as well as the fact that the transform between the two images is not rigid (the spine curves differently in both images). The bottom rows show the final contour, which has captured the boundary of the vertebra in the high contrast MR, and also in the lower contrast CT image. This results best illustrates the power of our joint segmentation/registration.
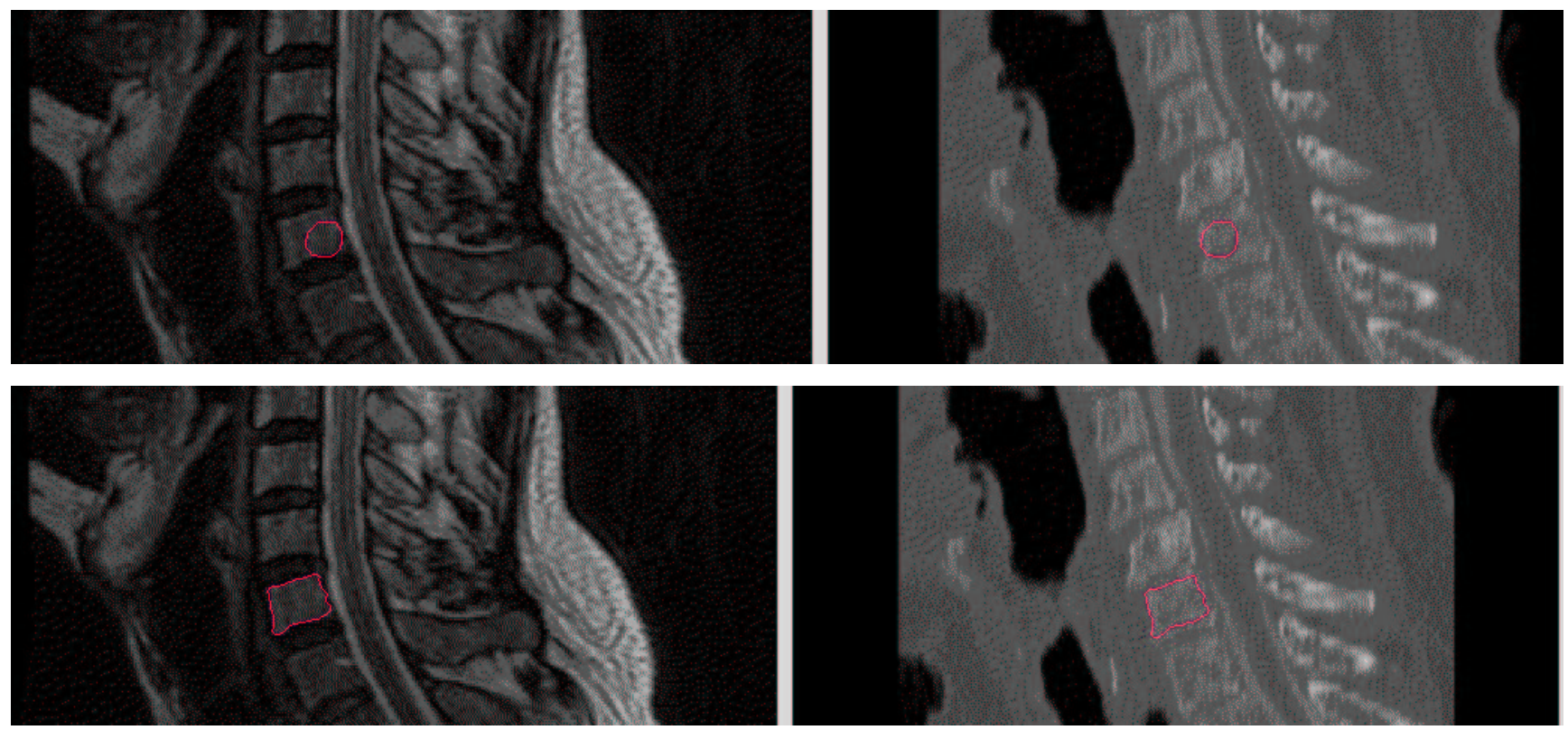
Figure 3: Registration/Segmentation of 3D MR and CT Images: Initial (left), intermediate (middle), and final (right) results. The top row shows the evolving surface. The bottom two rows shows cross-sections of the evolving surface overlaid on an $\mathrm{MR}$, and CT slice, respectively.

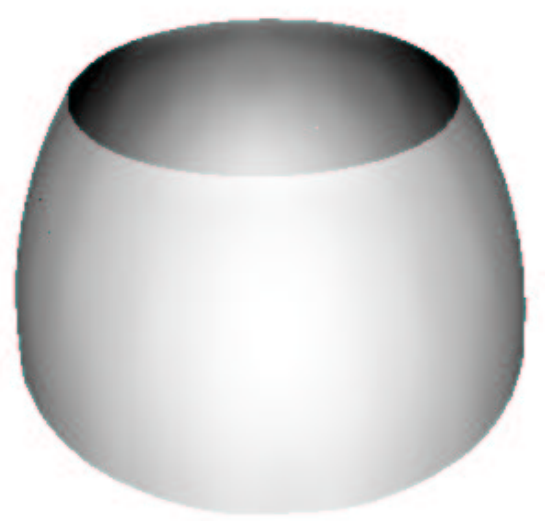

Initial Surface

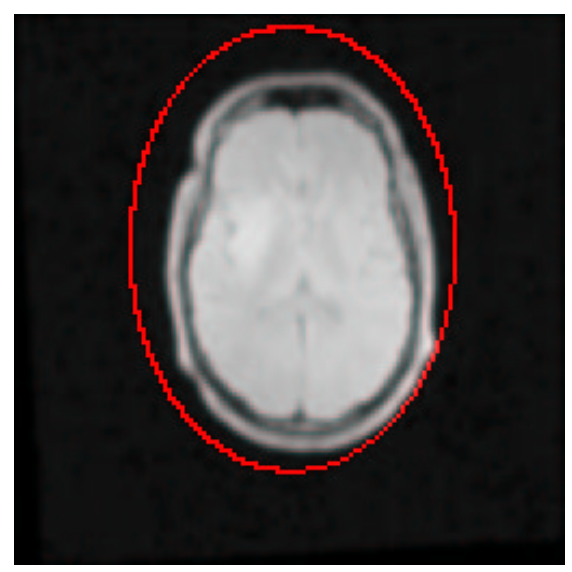

Cross-Section of Initial Surface

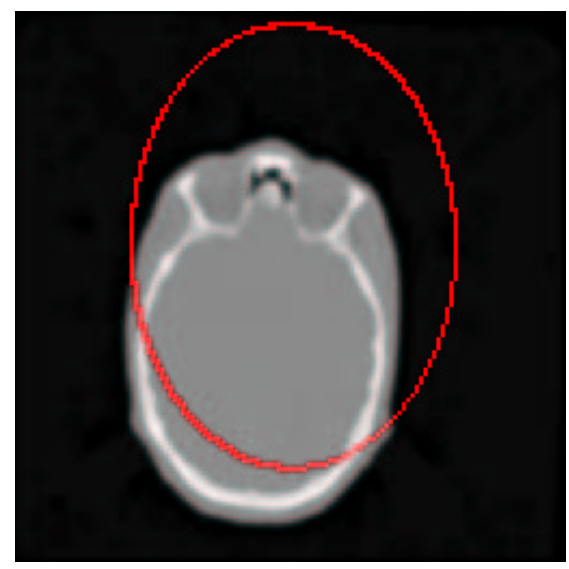

Cross-Section of Transformed Initial Surface

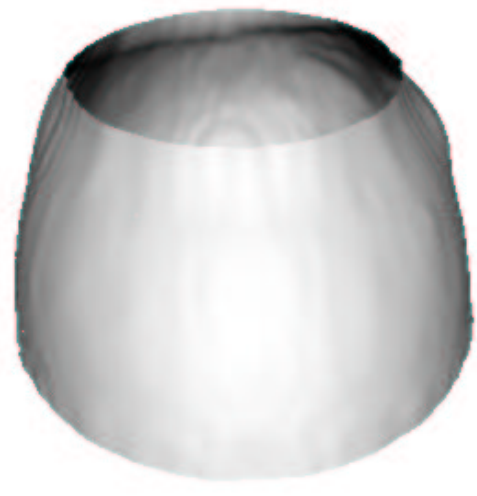

Intermediate Surface

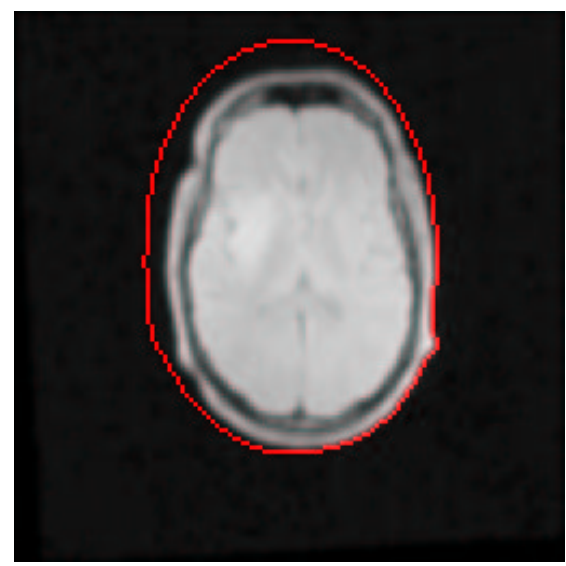

Cross-Section of Intermediate Surface

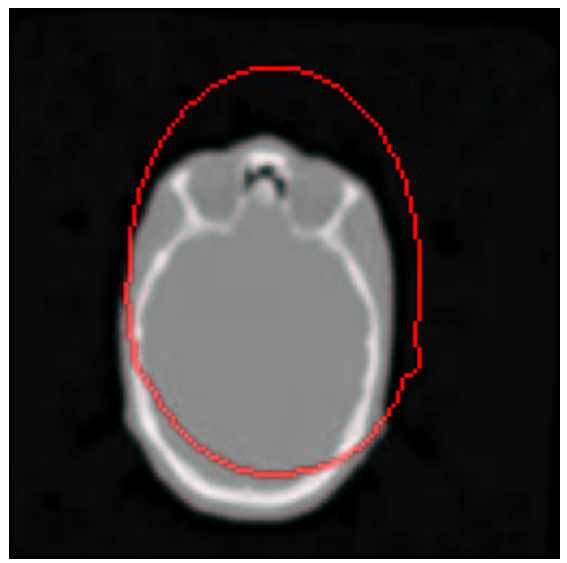

Cross-Section of Transformed Intermediate Surface

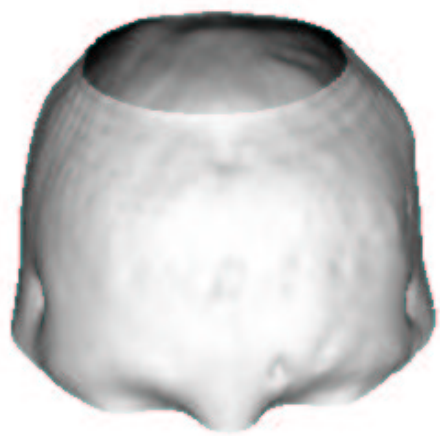

Final Surface

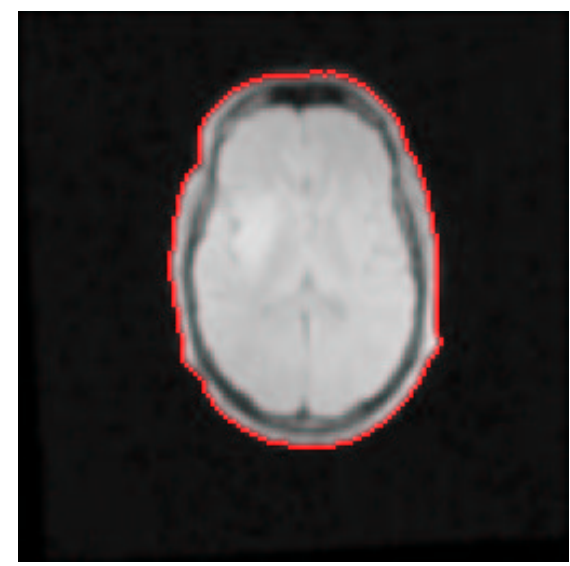

Cross-Section of Final Surface

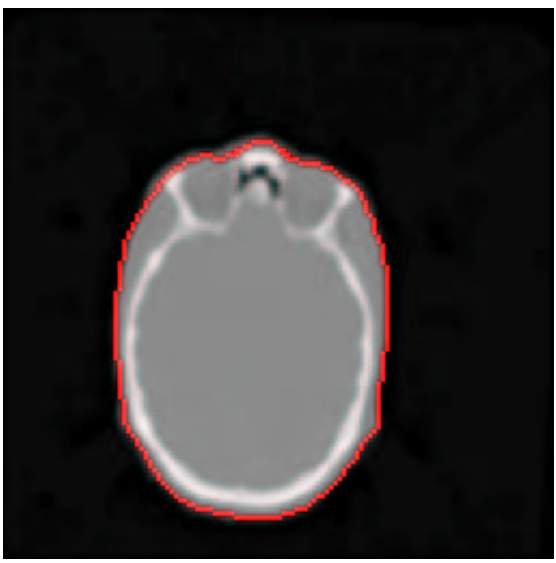

Cross-Section of Transformed Final Surface 